DFTT 22/95

CBPF-NF-17/95

\title{
The Role of the Central Element in the Quantum Algebra Underlying the Twisted XXZ Chain
}

\author{
by \\ M.R-Monteiro ${ }^{\dagger, \Delta, a}$, I. Roditi ${ }^{\Delta, b}$, L.M.C.S. Rodrigues ${ }^{\Delta, c}$, and S. Sciuto ${ }^{\dagger, d}$ \\ †Dipartimento di Fisica Teorica dell'Universitá di Torino \\ and Sezione di Torino dell'INFN, Via Pietro Giuria 1, \\ 10125, Torino, Italy \\ ${ }^{\Delta}$ Centro Brasileiro de Pesquisas Físicas - CBPF \\ Rua Dr. Xavier Sigaud, 150 \\ 22290-180, Rio de Janeiro, RJ, Brasil
}

e-mail:
(a)mmont@CBPFSU1.CAT.CBPF.BR
(b) Roditi@CBPFSU1.CAT.CBPF.BR
(c) Lígia@CBPFSU1.CAT.CBPF.BR 
(d) Sciuto@TO.INFN.IT 


\begin{abstract}
We study the relationship among the XXZ Heisenberg model and three models obtained from it by various transformations. In particular, we emphasize the role of a non trivial central element $t^{Z}$ in the underlying algebra and its relationship with the twisted boundary conditions, $S_{N+1}^{ \pm}=t^{ \pm N} S_{1}^{ \pm}$.
\end{abstract}

Key-words: Quantum Groups; Quantum Spin Chains; Integrable Models. 
The purpose of this letter is to study the relationship among three XXZ-like models: two of them are obtained from the periodic spin-1/2 XXZ chain [1] by performing De Vega [2] and Reshetikhin [3] like transformations in the $L$ and $R$ matrices such that the transformed matrices still obey the Yang-Baxter [4, 5] and the Fundamental Commutation Relations (FCR) [6-8] and the third one is the XXZ-like model studied in [9]. We find that the Hamiltonian with twisted boundary conditions comes out when the algebra contains a non-trivial central element, independently of the presence of the second parameter of deformation in the commutation relations.

We start briefly reviewing a few well-known elements of the Quantum Inverse Scattering Method (QISM) [6-8] applied to the periodic quantum spin-1/2 XXZ chain. The Hamiltonian describes a system of $N$ nearest neighbour interacting particles of spin-1/2,

$$
H=\sum_{\ell=1}^{N}\left(S_{\ell}^{+} S_{\ell+1}^{-}+S_{\ell}^{-} S_{\ell+1}^{+}+\Delta S_{\ell}^{3} S_{\ell+1}^{3}\right), \quad\left(\vec{S}_{N+1} \equiv \vec{S}_{1}\right)
$$

with $S^{ \pm}=S^{1} \pm i S^{2}, \vec{S}=\frac{1}{2} \vec{\sigma}$ and $\vec{\sigma}=\left(\sigma^{1}, \sigma^{2}, \sigma^{3}\right)$ the Pauli matrices, and $\Delta$ the anisotropy. $H$ acts on the $2^{N}$-dimensional Hilbert space,

$$
\mathcal{H}=\prod_{\ell=1}^{N} \otimes \eta_{\ell}
$$

where $\eta_{\ell} \equiv \mathbb{C}^{2}$, from now on called the internal space of the site " $\ell$ ".

In the QISM one introduces an auxiliary problem with the help of the so-called Lax operator (belonging to End $\left(V \otimes \eta_{\ell}\right)$ with $V \equiv \mathbb{C}^{2}$ the auxiliary space) which in this model is

$$
L_{\ell}(\lambda)=\left(\begin{array}{ll}
\operatorname{sh}\left[\gamma\left(\lambda+S_{\ell}^{3}\right)\right] & i \sin \gamma S_{\ell}^{-} \\
i \sin \gamma S_{\ell}^{+} & \operatorname{sh}\left[\gamma\left(\lambda-i S_{\ell}^{3}\right)\right]
\end{array}\right)
$$

$\lambda$ is a complex number called spectral parameter and $\gamma$ is a parameter associated to the anisotropy, $\Delta$, of the Hamiltonian according to

$$
\Delta=\frac{q+q^{-1}}{2}
$$

where $q=e^{i \gamma}$.

Associated to $L_{\ell}(\lambda)$ one introduces an invertible matrix $R(\lambda)$ belonging to End $(V \otimes V)$ 
which in this case is

$$
R(\lambda)=\left(\begin{array}{cccc}
a(\lambda) & 0 & 0 & 0 \\
0 & c(\lambda) & b(\lambda) & 0 \\
0 & b(\lambda) & c(\lambda) & 0 \\
0 & 0 & 0 & a(\lambda)
\end{array}\right)
$$

where

$$
a(\lambda)=\operatorname{sh}[\gamma(\lambda+i)], b(\lambda)=i \sin \gamma, c(\lambda)=\operatorname{sh} \gamma \lambda
$$

$R(\lambda)$ satisfies the Yang-Baxter equation

$$
R_{12}\left(\lambda_{12}\right) R_{13}\left(\lambda_{13}\right) R_{23}\left(\lambda_{23}\right)=R_{23}\left(\lambda_{23}\right) R_{13}\left(\lambda_{13}\right) R_{12}\left(\lambda_{12}\right)
$$

with $\lambda_{i j}=\lambda_{i}-\lambda_{j}$, and $L_{\ell}(\lambda)$ obeys the FCR

$$
R_{12}\left(\lambda_{12}\right) L_{1 \ell}\left(\lambda_{1}\right) L_{2 \ell}\left(\lambda_{2}\right)=L_{2 \ell}\left(\lambda_{2}\right) L_{1 \ell}\left(\lambda_{1}\right) R_{12}\left(\lambda_{12}\right)
$$

In (7) and (8)

$$
R_{12} \equiv \sum_{i} a_{i} \otimes b_{i} \otimes \mathbb{1}, R_{13} \equiv \sum_{i} a_{i} \otimes \mathbb{1} \otimes b_{i}, R_{23} \equiv \sum_{i} \mathbb{1} \otimes a_{i} \otimes b_{i}
$$

with the $R$ matrix written as

$$
R(\lambda)=\sum_{i} a_{i} \otimes b_{i}
$$

and the additional indices, 1 and 2 , in the $L_{\ell}$ matrix in (8) follow

$$
L_{1 \ell} \equiv L_{\ell} \otimes \mathbb{1} \quad, \quad L_{2 \ell} \equiv \mathbb{1} \otimes L_{\ell}
$$

We notice that comparing (7) and (8) for the case under consideration ((3), (5) and (6)), one has

$$
L_{1 \ell}(\lambda)=R_{1 \ell}(\lambda-i / 2)
$$

where the third auxiliary space is identified as the internal " $\ell$ " space.

For the vertex-type models that we are considering, a local Hamiltonian can be obtained as:

$$
H=\sum_{\ell=1}^{N} H_{\ell, \ell+1}=\left.C \frac{d \ln \tau}{d \lambda}\right|_{\lambda=i / 2}
$$

with $C$ a constant and

$$
\tau=\operatorname{Tr}_{0}\left(L_{0 N} L_{0 N-1} \cdots L_{01}\right)
$$


where the trace is over the auxiliary space denoted by "0". Thanks to the property (12) and to $R(0)=P$, with $P$ the permutation operator in the tensor product space under consideration, one can write

$$
H_{\ell, \ell+1}=\left.C \frac{d}{d \lambda}(P R)_{\ell, \ell+1}\right|_{\lambda=0}
$$

and with $R$ given in (5), one gets the Hamiltonian (1), apart from an additive constant.

In order to study the algebraic structure, after a similarity transformation on (7) and (8) one can factor out the $\lambda$ dependence in $R$ and $L[7$

$$
\begin{aligned}
L_{\ell}(\lambda) & =\frac{1}{2}\left(q^{-i \lambda} L^{+}-q^{i \lambda} L^{-}\right) \\
R(\lambda) & =\frac{1}{2}\left(q^{-i \lambda} R-q^{i \lambda} R^{\prime}\right)
\end{aligned}
$$

where

$$
L^{+}=\left(\begin{array}{cc}
q^{S^{3}} & \Omega S^{-} \\
0 & q^{-S^{3}}
\end{array}\right) \quad, \quad L^{-}=\left(\begin{array}{rr}
q^{-S^{3}} & 0 \\
-\Omega S^{+} & q^{S^{3}}
\end{array}\right)
$$

and

$$
R=\left(\begin{array}{llll}
q & 0 & 0 & 0 \\
0 & 1 & \Omega & 0 \\
0 & 0 & 1 & 0 \\
0 & 0 & 0 & q
\end{array}\right) \quad, \quad R^{\prime}=\left(\begin{array}{llll}
q^{-1} & 0 & 0 & 0 \\
0 & 1 & 0 & 0 \\
0 & -\Omega & 1 & 0 \\
0 & 0 & 0 & q^{-1}
\end{array}\right)
$$

with $\Omega=q-q^{-1}$, and $R, R^{\prime}$ satisfying

$$
\begin{aligned}
& P R P R^{\prime}=\mathbb{1}, \\
& R-R^{\prime}=\Omega P,
\end{aligned}
$$

$P$ being the permutation operator on the tensor product space $V \otimes V$.

Substituting (16) in the FCR one gets seven equations; it can be shown that due to (19) only three of them are independent and they can be chosen as:

$$
\begin{aligned}
& R_{12} L_{1 \ell}^{\varepsilon} L_{2 \ell}^{\varepsilon}=L_{2 \ell}^{\varepsilon} L_{1 \ell}^{\varepsilon} R_{12} \quad(\varepsilon= \pm) \\
& R_{12} L_{1 \ell}^{+} L_{2 \ell}^{-}=L_{2 \ell}^{-} L_{1 \ell}^{+} R_{12}
\end{aligned}
$$

Let us forget for a moment that the $\vec{S}$ are related to the Pauli matrices and consider them as abstract elements of an algebra; then equations (20) reduce to

$$
\begin{aligned}
{\left[S^{3}, S^{ \pm}\right] } & = \pm S^{ \pm} \\
{\left[S^{+}, S^{-}\right] } & =\frac{q^{2 S^{3}}-q^{-2 S^{3}}}{q-q^{-1}} \equiv\left[2 S^{3}\right]_{q} .
\end{aligned}
$$


which are the commutation relations of the quantum algebra $S L_{q}(2)$ [10, 11. It is obvious that equations (21) are trivially satisfied in the spin-1/2 representation of $S L(2)$ since in this case $S^{3}=0, \pm 1 / 2$ and $[n]_{q}=n$ for $n=0, \pm 1$, with $[x]_{q} \equiv \frac{q^{x}-q^{-x}}{q-q^{-1}}$.

The co-structure is obtained by analysing the product for different internal spaces of two $L^{\varepsilon}$ operators (as before, the matrices $\vec{S}$ are again taken as arbitrary elements of an algebra) defined in the same auxiliary space denoted by "0",

$$
\Delta\left(L^{ \pm}\right) \equiv L_{0 m}^{ \pm} L_{0 \ell}^{ \pm}
$$

which reduces to

$$
\begin{aligned}
\Delta q^{ \pm S^{3}} & =q^{ \pm S^{3}} \otimes q^{ \pm S^{3}} \\
\Delta S^{ \pm} & =q^{S^{3}} \otimes S^{ \pm}+S^{ \pm} \otimes q^{-S^{3}}
\end{aligned}
$$

Since $\Delta\left(L^{ \pm}\right)$satisfies the $F C R$ (eq.20), $\Delta q^{ \pm S^{3}}$ and $\Delta S^{ \pm}$given in (23) satisfy (21) nontrivially.

The co-product defined in (22) is non co-commutative, i.e.,

$$
\Delta^{\prime}\left(L^{ \pm}\right) \equiv L_{0 \ell}^{ \pm} L_{0 m}^{ \pm} \neq \Delta\left(L^{ \pm}\right)
$$

but one can show that there is an invertible matrix $\tilde{R} \in$ End $\left(\eta_{\ell} \otimes \eta_{m}\right)$ such that

$$
\tilde{R}_{m \ell} L_{0 m}^{ \pm} L_{0 \ell}^{ \pm}=L_{0 \ell}^{ \pm} L_{0 m}^{ \pm} \tilde{R}_{m \ell}
$$

Using $L_{21}^{+}(q)=L_{12}^{-}\left(q^{-1}\right)$, which holds in the spin-1/2 representation, $R_{21}(q)=R_{12}^{\prime}\left(q^{-1}\right)$ and (19), one easily checks that

$$
\tilde{R}_{m \ell}=P R_{m \ell} P
$$

with $P$ the permutation operator in the tensor product space $\eta_{\ell} \otimes \eta_{m}$. Defining $\tilde{R}(\lambda)=$ $\frac{1}{2}\left(q^{-i \lambda} \tilde{R}-q^{i \lambda} \tilde{R}^{\prime}\right)$ with $P \tilde{R} P \tilde{R}^{\prime}=\mathbb{1}$, one easily checks that $\tilde{R}(\lambda)=P \tilde{R}(\lambda) P$ and that the Yang-Baxter equation (7) can be rewritten as:

$$
\tilde{R}_{12}\left(\lambda_{12}\right) \tilde{R}_{13}\left(\lambda_{13}\right) \tilde{R}_{23}\left(\lambda_{23}\right)=\tilde{R}_{23}\left(\lambda_{23}\right) \tilde{R}_{13}\left(\lambda_{13}\right) \tilde{R}_{12}\left(\lambda_{12}\right)
$$

In the case under consideration $((5-6)), \tilde{R}(\lambda)=R(\lambda)$.

We are now going to analyse further deformed structures starting from the XXZ spin$1 / 2$ chain we have just presented. A new complex parameter $t$ is introduced through the transformations:

$$
g_{1}=t^{S^{3} \otimes Z} \quad, \quad g_{2}=t^{Z \otimes S^{3}}
$$


where $Z$ is a new operator; when acting on the auxiliary space or on the physical space of a single site, the algebra generated by $(\vec{S}, Z)$ will be taken in its fundamental representation; thus, unless stated otherwise,

$$
Z=\frac{1}{2} \mathbb{1} \quad, \quad \vec{S}=\frac{1}{2} \vec{\sigma}
$$

In this case, it is easy to check that

$$
\left[R_{12}(\lambda), g_{1} g_{2}\right]=0
$$

Using $g_{1}$ and $g_{2}$ we also define

$$
F_{12}=g_{1} g_{2}^{-1}=t^{S^{3} \otimes Z-Z \otimes S^{3}}
$$

which satisfies

$$
\begin{aligned}
& P F_{12} P F_{12}=\mathbb{1} \\
& F_{12} F_{13} F_{23}=F_{23} F_{13} F_{12}
\end{aligned}
$$

The XXZ chain will be denoted by case A. Using $R(\lambda), g$ and $F$ and with the help of De Vega [2] and Reshetikhin [3] type transformations we shall build other three possible cases:

CASE A:

$$
R^{A}(\lambda)=R(\lambda) ; L_{1 \ell}^{A}(\lambda)=R_{1 \ell}^{A}(\lambda-i / 2) ; \tilde{R}^{A}(\lambda)=P R^{A}(\lambda) P
$$

\section{CASE B:}

$$
R^{B}(\lambda)=R^{A}(\lambda) ; L_{1 \ell}^{B}(\lambda)=g_{1} L_{1 \ell}^{A}(\lambda) g_{1} ; \tilde{R}^{B}(\lambda)=F^{-1} \tilde{R}^{A}(\lambda) F^{-1} ;
$$

\section{CASE C:}

$$
R^{C}(\lambda)=F R^{A}(\lambda) F ; L_{1 \ell}^{C}(\lambda)=g_{\ell}^{-1} L_{1 \ell}^{A} g_{\ell}^{-1} ; \tilde{R}^{C}(\lambda)=\tilde{R}^{A}(\lambda) ;
$$

\section{CASE D:}

$$
R^{D}(\lambda)=R^{C}(\lambda) ; L_{1 \ell}^{D}(\lambda)=R_{1 \ell}^{D}(\lambda-i / 2)=F L_{1 \ell}^{A}(\lambda) F ; \tilde{R}^{D}(\lambda)=\tilde{R}^{B}(\lambda) ;
$$


we notice that for $g_{\ell}$ in (35) we take as auxiliary space the first vectorial space and as the " $\ell$ " internal space the second vectorial space. Thus $g_{\ell} \equiv g_{2}$ where $g_{2}$ is defined in (28) with the second vectorial space taken as the internal " $\ell$ " space.

As previously discussed for the case A, it can easily be checked for the cases B,C and D, using (30), that $L^{\bullet}, R^{\bullet}$ and $\tilde{R}^{\bullet}(\bullet=\mathrm{A}, \mathrm{B}, \mathrm{C}$ or D) satisfy (7), (8), (25), (27) and looking at eqs. (33-36) one immediately realizes that $R^{\bullet}(0)=P$, since $F$ satisfies (32).

The case $\mathrm{B}$ is an example of the procedure described in [2] since it preserves the $R$ matrix, transforming the $L$ matrix. The matrix $\tilde{R}^{B}$ is related to $\tilde{R}^{A}$ by a Reshetikhin transformation [3]; thus the de Vega transformation on the matrix $L$ can be seen as an application of the Reshetikhin theorem at the level of QISM.

Instead the case $\mathrm{C}$ is got from the case $\mathrm{A}$ by the dual of the transformation sending A into B; we shall call the latter a "de Vega transformation" and the former a "dual Reshetikhin transformation." These two transformations are related to each other by the interchange of the role of the auxiliary space and of the site space; this is particularly clear if one compares $L_{1 \ell}^{B}=g_{1} L_{1 \ell}^{A} g_{1}$ with $L_{1 \ell}^{C}=g_{\ell}^{-1} L_{1}^{A} g_{\ell}^{-1}$.

Noticing that (34-36) imply

$$
\begin{aligned}
L_{1 \ell}^{D} & =g_{\ell}^{-1} L_{1 \ell}^{B} g_{\ell}^{-1}, \\
L_{1 \ell}^{D} & =g_{1} L_{1 \ell}^{C} g_{1},
\end{aligned}
$$

one realizes that the case D [9] can be obtained either from B by applying a dual Reshetikhin transformation or from $\mathrm{C}$ through a de Vega transformation.

We are now going to show that the cases connected by a dual Reshetikhin transformation have the same Hamiltonian. In cases C and D using (14), (35) and (37) we get

$$
\begin{aligned}
\tau^{C} & =G^{-1} \tau^{A} G^{-1} \\
\tau^{D} & =G^{-1} \tau^{B} G^{-1}
\end{aligned}
$$

with

$$
G=\prod_{\ell=1}^{N} g_{\ell}=t^{\frac{1}{2} S_{\text {total }}^{3}}
$$

As

$$
H=\left.C \tau^{-1} \frac{d \tau}{d \lambda}\right|_{\lambda=i / 2}
$$


using $\left[g_{\ell} g_{\ell+1}, R_{\ell, \ell+1}\right]=0$, which in the case we are considering means that $H_{\ell, \ell+1}^{A}$ is invariant under a simultaneous rotation of the sites $\ell$ and $\ell+1$ around the $z$-axis, we obtain

$$
\begin{aligned}
& H^{C}=G H^{A} G^{-1}=H^{A} \\
& H^{D}=G H^{B} G^{-1}=H^{B} .
\end{aligned}
$$

The Hamiltonian can be directly written in terms of $R_{\ell, \ell+1}$ (eq. 15) only in the cases A and $\mathrm{D}$, where the identity $L_{1 \ell}(\lambda)=R_{1 \ell}(\lambda-i / 2)$ holds. However, it is amusing to observe that, since $P R^{A}=\tilde{R}^{A} P=\tilde{R}^{C} P$ and $P R^{D}=\tilde{R}^{D} P=\tilde{R}^{B} P$, in all cases we can write the Hamiltonian as

$$
H^{\bullet}=\sum_{\ell=1}^{N} H_{\ell, \ell+1}^{\bullet} \quad, H_{\ell, \ell+1}^{\bullet}=\left.C \frac{d \tilde{R}_{\ell, \ell+1}^{\bullet}}{d \lambda}\right|_{\lambda=0} P
$$

with $\bullet=\mathrm{A}, \mathrm{B}, \mathrm{C}$ or $\mathrm{D}$, which has the same form as the Hamiltonian of a spin- $S$ system. The dependence on $\tilde{R}$ is expected since this matrix controls the non-cocommutativity of the coproduct, which is the relevant element for the construction of the Hamiltonian. Furthermore, the dependence of (42) on $\tilde{R}$ makes evident the conclusions expressed in (41) by just looking at the relations for the various $\tilde{R}$ in (33-36).

In the cases $\mathrm{B}$ and $\mathrm{D}$, using the explicit expression

$$
R^{D}=\left(\begin{array}{llll}
a & 0 & 0 & 0 \\
0 & t c & b & 0 \\
0 & b & t^{-1} c & 0 \\
0 & 0 & 0 & a
\end{array}\right)
$$

and eq. (15) (or (42)) we get 12, 9, 13

$$
H_{\ell, \ell+1}^{D}=g_{\ell+1}^{2} H_{\ell, \ell+1}^{A} g_{\ell+1}^{-2}=\left(t^{-1} S_{\ell}^{+} S_{\ell+1}^{-}+t S_{\ell}^{-} S_{\ell+1}^{+}+2 \cos \gamma S_{\ell}^{3} S_{\ell+1}^{3}\right),
$$

apart from an additive constant. The similarity transformation generated by

$$
K=\exp \left[i \alpha \sum_{\ell=1}^{N}(\ell-1) S_{\ell}^{3}\right]
$$

with $t=e^{i \alpha}$, takes $H^{D}$ to $\tilde{H}^{D}=K^{-1} H^{D} K$, that is [9],

$$
\begin{aligned}
\tilde{H}^{D} & =\sum_{\ell=1}^{N-1}\left(S_{\ell}^{+} S_{\ell+1}^{-}+S_{\ell}^{-} S_{\ell+1}^{+}+2 \cos \gamma S_{\ell}^{3} S_{\ell+1}^{3}+2 \cos \gamma S_{N}^{3} S_{1}^{3}+\right. \\
& \left.+t^{-N} S_{N}^{+} S_{1}^{-}+t^{N} S_{N}^{-} S_{1}^{+}\right)
\end{aligned}
$$


which is the well-known Hamiltonian for the XXZ chain with twisted boundary conditions $\left(S_{N+1}^{ \pm} \equiv t^{ \pm N} S_{1}^{ \pm}\right)$14.

In summary, the Hamiltonians for the cases A,B,C and D are:

$$
\begin{aligned}
& H^{C}=H^{A}=H_{X X Z} \\
& H^{D}=H^{B}=H_{X X Z}^{\text {twisted }} .
\end{aligned}
$$

Let us now consider the underlying algebraic structure in cases B,C and D. For the case B the $L$ matrix is given by $L_{1 \ell}^{B}=g_{1} L_{1 \ell}^{A} g_{1}$ with:

$$
g_{1}=t^{S^{3} \otimes Z_{\ell}}=\left(\begin{array}{ll}
t^{\frac{1}{2} Z_{\ell}} & 0 \\
0 & t^{-\frac{1}{2} Z_{\ell}}
\end{array}\right)
$$

which leads, after factoring out the spectral parameter, to

$$
L_{+}^{B}=\left(\begin{array}{ll}
q^{S^{3}} t^{Z} & \Omega S^{-} \\
0 & q^{S^{3}} t^{-Z}
\end{array}\right) \quad, \quad L_{-}^{B}=\left(\begin{array}{ll}
q^{-S^{3}} t^{Z} & 0 \\
-\Omega S^{+} & q^{S^{3}} t^{-Z}
\end{array}\right)
$$

where $Z$ as well as $\vec{S}$ are taken in an arbitrary representation. The $R$-matrix is the same as in case $\mathrm{A}$ and thus the equations

$$
\begin{aligned}
& R_{12}^{B} L_{1 \ell}^{ \pm B} L_{2 \ell}^{ \pm B}=L_{2 \ell}^{ \pm B} L_{1 \ell}^{ \pm B} R_{12}^{B} \\
& R_{12}^{B} L_{1 \ell}^{+B} L_{2 \ell}^{-B}=L_{2 \ell}^{-B} L_{1 \ell}^{+B} R_{12}^{B}
\end{aligned}
$$

reduce to

\section{CASE B:}

$$
\begin{aligned}
{\left[S^{3}, S^{ \pm}\right] } & = \pm S^{ \pm} \\
{\left[S^{+}, S^{-}\right] } & =\left[2 S^{3}\right]_{q} \\
{[Z, \vec{S}] } & =0 .
\end{aligned}
$$

The co-structure is, as usual, obtained by considering the product of two $L^{B}$ acting on two internal spaces (see eq. (22)); we find

\section{CASE B:}

$$
\begin{aligned}
\Delta S^{3} & =S^{3} \otimes \mathbb{1}+\mathbb{1} \otimes S^{3} \\
\Delta Z & =Z \otimes \mathbb{1}+\mathbb{1} \otimes Z \\
\Delta S^{ \pm} & =q^{S^{3}} t^{\mp Z} \otimes S^{ \pm}+S^{ \pm} \otimes q^{-S^{3}} t^{ \pm Z}
\end{aligned}
$$


that is, we have $S L_{q}(2)$ with a central element $t^{Z}$ [15, 16]. Notice that this central element appears in a non-trivial way in the co-product.

In case C, we have $L_{1 \ell}^{C}=g_{\ell}^{-1} L_{1 \ell}^{A} g_{\ell}^{-1}$ with $g_{\ell}=t^{Z \otimes S_{\ell}^{3}}=1 \otimes t^{\frac{1}{2}} S_{\ell}^{3}$ which, after factoring out the spectral parameter, gives.

$$
L_{+}^{C}=\left(\begin{array}{ll}
\left(q t^{-1}\right)^{S_{3}} & \Omega S^{-} \\
0 & (q t)^{-S_{3}}
\end{array}\right) \quad, L_{-}^{C}=\left(\begin{array}{ll}
(q t)^{-S_{3}} & 0 \\
-\Omega S^{+} & \left(q t^{-1}\right)^{S_{3}}
\end{array}\right),
$$

and

$$
R^{C}=F R^{A} F=\left(\begin{array}{rrrr}
q & 0 & 0 & 0 \\
0 & t & \Omega & 0 \\
0 & 0 & t^{-1} & 0 \\
0 & 0 & 0 & q
\end{array}\right) .
$$

Thus, by a similar procedure as in the previous case we have for the algebra

\section{CASE C:}

$$
\begin{aligned}
{\left[S^{3}, S^{ \pm}\right] } & = \pm S^{ \pm} \\
t^{-1} S^{+} S^{-}-t S^{-} S^{+} & =t^{-2 S_{3}}\left[2 S_{3}\right]_{q}
\end{aligned}
$$

and for the co-product

\section{CASE C:}

$$
\begin{aligned}
\Delta S^{3} & =S^{3} \otimes \mathbb{1}+\mathbb{1} \otimes S^{3} \\
\Delta S^{ \pm} & =\left(q t^{-1}\right)^{S^{3}} \otimes S^{ \pm}+S^{ \pm} \otimes(q t)^{-S^{3}}
\end{aligned}
$$

which is the two-parameter deformed $S L_{q, t}(2)$ algebra [3, 17, 18. As noted in ref. [16] the structure and costructure of $S L_{q, t}(2)$ given respectively in (55) and (56), are actually isomorphic to $S L_{q}(R)(21,23)$ as one can easily check by defining

$$
\tilde{S}_{3}=S_{3} \quad, \quad \tilde{S}^{ \pm}=S^{ \pm} t^{S_{3}}
$$

The case $\mathbf{D}$ follows similarly. In this case we have $L_{1 \ell}^{D}=g_{\ell}^{-1} L_{1 \ell}^{B} g_{\ell}^{-1}$ which reduces to:

$$
L_{+}^{D}=\left(\begin{array}{ll}
\left(q t^{-1}\right)^{S^{3}} t^{Z} & \Omega S^{-} \\
0 & (q t)^{-S^{3}} t^{-Z}
\end{array}\right) \quad, \quad L_{-}^{D}=\left(\begin{array}{ll}
(q t)^{-S^{3}} t^{Z} & 0 \\
-\Omega S^{+} & \left(q t^{-1}\right)^{S^{3}} t^{-Z}
\end{array}\right)
$$


and $R^{D}=R^{C}$. With this in hand, as in the previous cases we have

\section{CASE D:}

$$
\left[S^{3}, S^{ \pm}\right]= \pm S^{ \pm} ; t^{-1} S^{+} S^{-}-t S^{-} S^{+}=t^{-2 S^{3}}\left[2 S^{3}\right]_{q} ;[Z, \vec{S}]=0
$$

and for the co-product:

\section{CASE D:}

$$
\begin{aligned}
\Delta S^{3} & =\mathbb{1} \otimes S^{3}+S^{3} \otimes \mathbb{1} \\
\Delta Z & =Z \otimes \mathbb{1}+\mathbb{1} \otimes Z \\
\Delta S^{ \pm} & =\left(q t^{-1}\right)^{S^{3}} t^{\mp Z} \otimes S^{ \pm}+S^{ \pm} \otimes(q t)^{-S^{3}} t^{ \pm Z}
\end{aligned}
$$

which corresponds to the $S L_{q, t}(2)$ algebra with a central element $t^{Z}$; once more the central element appears in a non trivial way in the co-product.

By the transformation (57) eqs. (59-60) are sent into the case B eqs. (51-52). By comparing the cases related by a "De Vega transformation" $(A \rightarrow B$ and $C \rightarrow D)$ one easily realizes that the identities

$$
\begin{aligned}
& \Delta^{B}=F \Delta^{A} F^{-1} \\
& \Delta^{D}=F \Delta^{C} F^{-1}
\end{aligned}
$$

hold and that the commutation relations do not change, in agreement with the Reshetikhin theorem [3], apart from the enlargement of the algebra due to the introduction of the central element $Z$.

The Bethe ansatz equations can be easily computed and are those of the periodic XXZ chain in cases $A, C$ and those of the twisted XXZ chain in cases $B$ and $D$.

In summary, we have analysed three different XXZ like models. They are obtained by performing De Vega and dual Reshetikhin transformations, which are dual to each other, in the $L$ and $R$ matrices of the periodic spin-1/2 chain. We have shown that De Vega transformation introduces a central element, $t^{Z}$, in the underlying algebraic structure which gives rise to twisted boundary conditions, $S_{N+1}^{ \pm}=t^{ \pm N} S_{1}^{ \pm}$. These cases were here called $\mathrm{B}$ and $\mathrm{D}$. In cases $A$ and $C$ the is no central element in the underlying algebraic structure and these cases correspond to the periodic spin-1/2 XXZ chain. As the 
only consequence of the dual Reshetikhin transformation is the introduction of a second parameter in the commutation relations, without the presence of a non-trivial central element in the algebra, it does not change the Hamiltonian of the theory.

Moreover we have shown that in all the cases we have considered, the Hamiltonian can be written in an universal way in terms of $\tilde{R}$ (41), which, in our notation, is not the $R$ matrix entering into $F C R(R L L=L L R)$ but its counterpart in the dual algebra.

Finally, we remark that the present discussion can be generalized to any model with invariance properties analogous to eq. 30 . 


\section{References}

[1] E. Lieb, Phys. Rev. Lett. 18, (1967) 692; Phys. Rev. 162, (1968) 162; Phys. Rev. Lett. 18, (1967) 1046; Phys. Rev. Lett. 19, (1967) 108.

[2] H.J. De Vega, Nucl. Phys. B240 (1984) 495; Int. Jour. Mod. Phys. A4 (1989) 2371; H.J. De Vega and E. Lopes, Phys. Lett. B186 (1987) 180.

[3] N. Reshetikhin, Lett. Math. Phys. 20, (1990) 331.

[4] R. Baxter, Ann. of Phys. 70 (1972) 193; Ann. of Phys. 70 (1972) 323.

[5] C.N. Yang, Phys. Rev. Lett. 19 (1967) 1312.

[6] E. Sklyanin, L. Takhtajan and L. Faddeev, Teor. Matem. Fiz. 40 (1979) 194; L. Takhtajan and L.Faddeev, Russian Math. Surveys 34 (1979) 11; N. Reshetikhin, L. Takhtajan and L. Faddeev, Leningrad Math. J. 1 (1990) 193.

[7] L. Faddeev, in "New Problems, Methods and Techniques in Quantum Fielf Theory and Statistical Mechanics", edited by M. Rasetti, World Scientific Pub. (1990); "Algebraic Aspects of Bethe-Ansatz", preprint ITP-SB-94-11.

[8] For a review see: "Quantum Inverse Scattering Method and Correlation Functions" by V.E. Korepin, N.M. Bogoliubov and A.G. Izergin, Cambridge U.P. (1993).

[9] M.R-Monteiro, I. Roditi, L.M.C.S. Rodrigues and S. Sciuto, "The Quantum Algebraic Structure of the Twisted XXZ Chain", preprint CBPF-NF-054/94 and DFTT045/94, hep-th/9410144, to appear in Mod. Phys. Lett. A.

[10] P. Kulish and N. Reshetikhin, Zap. Nauch. Seminarov LOMI 101, (1981) 101; J. Sov. Math. 23 (1983) 2435.

[11] V. Drinfeld, Sov. Math. Dokl. (1985) 254; M. Jimbo, Lett. Math. Phys. 10, (1985) $63 ; 11$ (1986) 247.

[12] L. Hlavatý, "Solution to the YBE Corresponding to the XXZ Models in an External Magnetic Field", preprint E5-85-959, Dubna (1985); J. Math. Phys. A 27 (1994) 5645 . 
[13] J. Abad and M. Rios, "Integrable Spin Chain Associated to $\widehat{S L}_{q}(n)$ and $\widehat{S L}_{p, q}(n)$ ", preprint DFTUZ/94/21, hep-th/9410193.

[14] F. Alcaraz, U. Grimm and V. Rittenberg, Nucl Phys. B316 (1989) 735; V. Pasquier and H. Saleur, Nucl. Phys. B330, (1990), 523.

[15] C. Burdik and P. Hellinger, J. Phys. A25 (1992) L629.

[16] R. Chakrabarti and R. Jagannathan, J. Phys. A27 (1994) 2023.

[17] A. Sudbery, J. Phys. A23, (1990) L697; M. Takeuchi, Proc. Jap. Acad. 66, (1990) 112.

[18] C. Burdik and L. Hlavatý, J. Phys. A24, (1991) L165. 